\title{
Performance of point-of-care CD4 testing technologies in resource-constrained settings: a systematic review and meta- analysis
}

Minh D. PhamM.D., MPH ${ }^{1,2^{*}}$, Paul A. Agius ${ }^{1}$, Lorena Romero ${ }^{3}$, Peter McGlynn², David Anderson ${ }^{1,4}$, Suzanne M. Crowe ${ }^{1,5}$ and Stanley Luchters ${ }^{1,2,6}$

\begin{abstract}
Background: Point-of-care (POC) CD4 testing increases patient accessibility to assessment of antiretroviral therapy eligibility. This review evaluates field performance in low and middle-income countries (LMICs) of currently available POC CD4 technologies.

Methods: Eight electronic databases were searched for field studies published between January 2005 and January 2015 of six POC CD4 platforms: PointCare NOW ${ }^{\mathrm{TM}}$, Alere Pima ${ }^{\mathrm{TM}}$ CD4, Daktari ${ }^{\mathrm{TM}}$ CD4 Counter, CyFlow ${ }^{\circledR}$ CD4 miniPOC, BD FACSPresto ${ }^{\mathrm{TM}}$, and MyT4 ${ }^{\mathrm{TM}} \mathrm{CD}$ 4. Due to limited data availability, meta-analysis was conducted only for diagnostic performance of Pima at a threshold of 350 cells/ $\mu$ l, applying a bivariate multi-level random-effects modelling approach. A covariate extended model was also explored to test for difference in diagnostic performance between capillary and venous blood.

Results: Twenty seven studies were included. Published field study results were found for three of the six POC CD4 tests, 24 of which used Pima. For Pima, test failure rates varied from 2 to $23 \%$ across study settings. Pooled sensitivity and specificity were $0.92(95 \% \mathrm{Cl}=0.88-0.95)$ and $0.87(95 \% \mathrm{Cl}=0.85-0.88)$ respectively. Diagnostic performance by blood sample type (venous vs. capillary) revealed non-significant differences in sensitivity (0.94 vs 0 . 89 ) and specificity (0.86 vs 0.87), respectively in the extended model (Wald $x^{2}(2)=4.77, p=0.09$ ).

Conclusions: POC CD4 testing can provides reliable results for making treatment decision under field conditions in low-resource settings. The Pima test shows a good diagnostic performance at CD4 cut-off of $350 \mathrm{cells} / \mu \mathrm{l}$. More data are required to evaluate performance of POC CD4 testing using venous versus capillary blood in LMICs which might otherwise influence clinical practice.
\end{abstract}

Keywords: CD4, Point of care testing, Diagnostic accuracy, Systematic review, Meta-analysis

\section{Background}

Increased testing of those at risk of HIV infection and improving access to antiretroviral therapy (ART) particularly in the most HIV-affected regions is a recognized global strategy to end the AIDS epidemic. However, only 15.0 million of an estimated of 36.9

\footnotetext{
* Correspondence: minh.pham@burnet.edu.au

${ }^{1}$ Burnet Institute, 85 Commercial Road, Melbourne, VIC 3004, Australia ${ }^{2}$ Department of Epidemiology and Preventive Medicine, Faculty of Medicine Nursing and Health Science, Monash University, Melbourne, Australia

Full list of author information is available at the end of the article
}

million (40.6 \%) HIV-infected individuals have access to ART [1]. Whilst new evidence endorses treatment of all HIV-infected individuals [2, 3] and the World Health Organization (WHO) guideline supports treatment of all individuals regardless of CD4 count [4], the current practical goal, one that is likely to apply in most resource limited countries into the foreseeable future, is to achieve ART coverage of all HIV-infected adults with low CD4 cell count $(<350$ cells $/ \mu \mathrm{L})$ before expanding ART scale-up to people with higher CD4 cell counts. Furthermore, for patients who are presenting late to 
care, a CD4 count is required as a baseline measurement to identify the need for screening and prophylaxis for major opportunistic infections which are often associated with low CD4 count and increased risk of mortality. For treatment monitoring, CD4 count is important to assess CD4-related risk of toxicity to Nevirapine and, CD4 testing remains an important method to monitor patients who are on treatment in settings where access to viral load monitoring is still limited [5]. Thus, CD4 monitoring remains an essential and practical component of HIV care in the near future [6].

It has been known that barriers resulting in substantial losses to the continuum of HIV care include poor access to CD4 testing, particularly in disadvantaged and remote areas where laboratory-based CD4 testing by flow cytometry is not available $[7,8]$. Point-of-care (POC) testing is an effective strategy to overcome this challenge. Findings from a number of field studies show that POC $\mathrm{CD} 4$ testing can have a positive impact on the HIV continuum of care [9-15]. The use of POC CD4 in lower and middle income countries (LMICs) where resources for HIV care are most limited, is expected to produce greatest clinical and economic impacts from both patient and health system perspectives [16, 17]. With a number of POC CD4 technologies available or in the pipe-line [18], there is a need for consolidated evidence on the performance of different POC CD4 tests, particularly in LMICs, to inform decision-making related to selection of an appropriate test in field settings. A systematic review and a meta-analysis of test performance of CD4 count technologies showed that POC CD4 test is suitable for ART eligibility assessment at CD4 thresholds of 350 and 500 cells/ $\mu \mathrm{L}[19,20]$. However, these studies pooled data from both laboratory and field evaluations in low and high income countries. As there is evidence to suggest that performance of the test varies significantly across evaluation settings (laboratory vs clinic) in different countries [21, 22], the question remains whether these study findings are transferable, specifically to non-laboratory environments in LMICs. We, therefore, conducted a systematic review to assess the performance, acceptability and feasibility in non-laboratory field settings of currently available or prototype commercial POC CD4 tests in LMICs. Here, we report on "field performance" of different POC CD4 tests; findings on "acceptability, feasibility" will be reported elsewhere.

\section{Methods}

This systematic review was conducted according to a protocol developed using the preferred reporting items for systematic reviews and meta-analyses (PRISMA) statement [23].

\section{Literature search strategy}

The search strategy was designed to identify any studies describing POC CD4 tests. After an initial search for articles in Medline and Embase, an assessment of text words within the title and abstract and of the index terms used to describe these articles was conducted. A subsequent full search using clearly established search terms (see Additional file 1: Annex 1) was undertaken across included databases, and adapted as appropriate to the specifications for the respective databases: Medline, Embase, CENTRAL, Cinahl, PsycINFO, Biological Abstracts, and Scopus. Web of Science and conference databases were also searched to identify relevant studies. Reference lists of all identified reports and articles were searched for additional studies. Moreover, searches were conducted in grey literature resources such as conference websites (NLM Gateway, the British Library Conference and International AIDS Society and Conference on Retroviruses and Opportunistic Infections) and clinical trials websites. Hand-searching and reference checking of citations and reference lists was undertaken. Authors of relevant studies were contacted if insufficient data were published. Government reports, letters to editors, commentaries, editorials, non-peer reviewed articles and review articles were excluded. Studies conducted and funded by the manufacturer, if stated, were also excluded.

\section{Study selection}

Inclusion criteria were that studies needed to be published between January 2005 and January 2015, written in English language and conducted in LMICs (http:// data.worldbank.org/about/country-and-lending-groups). Studies conducted in both LMICs and non-LMICs were included if data from LMICs were presented separately. Further eligibility criteria were defined using PICO (participants, interventions, comparisons, outcomes) format [24]. Participants (P) included HIV positive, HIV negative and unknown HIV status persons aged $\geq 12$ months. For intervention (I), any of the following six commercially available POC CD4 testing platforms listed in the UNITAID "2014 HIV/AIDS Diagnosis Technology Landscape" report [18] were included: (1) PointCare NOW ${ }^{\mathrm{m}}$ (PointCare Technology Inc, Marlborough, MA, USA); (2) Pima ${ }^{\text {Tx }}$ CD4 (Alere Inc, Waltham, MA, USA); (3) Daktari $^{\text {Tin }}$ CD4 Counter (Daktari Diagnostics Inc, Cambridge MA, USA); (4) CyFlow ${ }^{\circ}$ CD4 miniPOC (Partec, Munich, Germany); (5) BD FACSPresto ${ }^{\mathrm{Tm}}$ (BD Biosciences, San Jose, CA, USA); and (6) MyT4 ${ }^{\text {ma }}$ CD4 Test (Zyomyx Inc, Fremont, CA, USA). Results of POC CD4 test needed to be compared $(\mathrm{C})$ to reference laboratorybased assays with outcomes $(\mathrm{O})$ containing diagnostic performance of POC CD4 test in field settings. All retrieved articles were checked for duplication; conference 
abstracts were excluded if duplicated with full-text articles. Titles, abstracts and summaries of identified records were screened for relevance. Retained records meeting the inclusion criteria were then examined in full text (Fig. 1).

\section{Data extraction and data analysis}

An electronic data extraction form was developed, pretested and finalized by consensus among authors. Data extraction was conducted by one reviewer and verified by the second using the data extraction form with $20 \%$ duplicate extraction. Quality of included studies was assessed using EPHPP tool for quantitative studies [25] and QUADAS tool for diagnostic accuracy studies [26].

Key measures used to evaluate performance of POC CD4 tests were: (1) failure rates defined as the percentage of the total number of tests performed with an invalid result such that tests did not provide results and/or results could not be read. (2) Sensitivity and specificity of index POC CD4 test at certain CD4 thresholds as compared to the predicate method. (3) Misclassification of index POC CD4 at certain CD4 thresholds. Misclassification is defined as the percentage of total HIVinfected individuals/blood samples tested with disagreement in results between POC CD4 and predicate method for the purpose of identifying ART eligibility at pre-specified CD4 threshold values of 200, 350 and 500 cells $/ \mu \mathrm{l}$; and (4) Difference in mean (bias) of CD4 counts and limit of agreement between index POC CD4 and predicate method. Bias included absolute and relative bias defined as the mean absolute and/or relative difference in $\mathrm{CD} 4$ count between the index POC CD4 and the predicate test. Limit of agreement was calculated as the mean difference \pm 1.96 standard deviation of the difference.
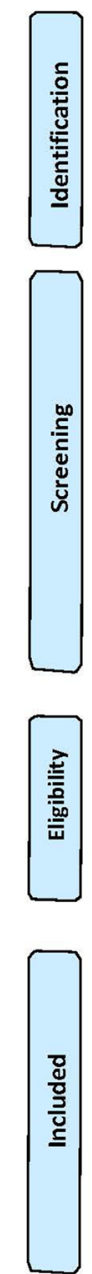

Two studies reported on PointCare NOW ${ }^{\mathrm{TM}}$ and one on MyT4 ${ }^{\mathrm{TM}}$ CD4. Eight studies reported on Pima but did not provide data required for the meta-analyses database searching
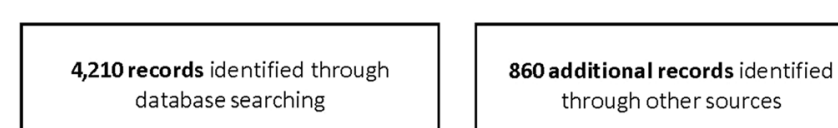
through other sources
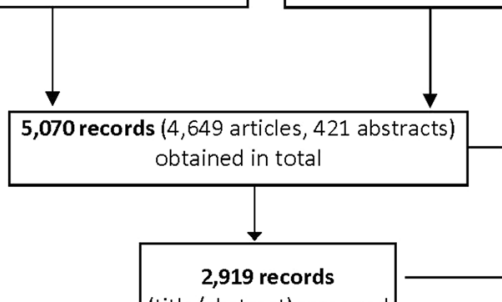

2,151 duplicated records removed

2,839 records excluded 2,919 records
(title/abstract) screened

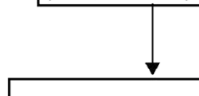

80 full-text (56 articles, 24 abstracts) assessed for eligibility

27 studies included in qualitative synthesis

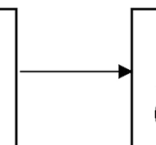

53 full-text excluded: editorials (5), reviews (2), study protocol (3), costing study (4 articles, 2 abstracts), systematic review (3), not field testing in LMICs ( 3 articles, 3 abstracts), no comparator (2), not outcomes of interest (2), use other POC tests ( 8 articles, 3 abstracts), abstracts duplicated with full text (4), abstracts do not provide full PICO information (9) 
A meta-analysis of diagnostic test accuracy (DTA) was conducted of the Alere Pima ${ }^{\text {тм }}$ CD4 (Pima) test through application of a bivariate multi-level random effects modeling approach [27], recommended for metaanalysis for Cochrane DTA reviews. The bivariate random-effects model accounts for both the correlation between study sensitivity and specificity estimates, and also unobserved between-study heterogeneity in test performance through specification of a multi-level bivariate normal regression approach. The bivariate model estimates sensitivity and specificity by modeling randomeffects across two levels; level 1 representing the withinstudy variability between sensitivity and specificity and level 2 representing heterogeneity in diagnostic performance of the index test across studies. Using the multilevel bivariate model; sensitivity, specificity, positive and negative likelihood ratios $( \pm \mathrm{LR})$ of the Pima test were estimated. In order to test for any difference in diagnostic performance across venous and capillary sample methods, the multi-level bivariate random-effects model was extended to include a covariate for blood sample type. Post-estimation Wald tests were used to test the joint (i.e. for sensitivity and specificity simultaneously) effect of blood sample type on diagnostic accuracy. In all multi-level analyses of pooled Pima test diagnostic performance, Huber/White variance estimation was used to provide appropriate standard errors in instances where multiple sets of diagnostic data were taken from a single study [28]. Diagnostic statistics (Cook's distance [using a 5 parameter model based cutoff $>2$ ] and standardized residual plots) from multi-level bivariate random-effect models were examined to assess pooled sensitivity and specificity estimates for outlier bias. Using Stata version 13.1 (Stata Corporation, TX, USA), both the user-written Stata program Midas [29] and author-written code using GLLAMM (Generalized Latent and Linear Mixed Modeling) [30] were used to provide key statistical output for pooled multi-level bivariate random-effect model analyses of the diagnostic performance of the Pima test. The user-written Stata program metandiplot [31] was used to plot the hierarchical summary receiver operating characteristic (HSROC) curve [32] from observed study sensitivity and specificity estimates. Statistical significance was assessed at $5 \%$ in all analyses.

\section{Results}

\section{Study characteristics}

The initial search, after removing duplicates, yielded 2,919 records, among which 27 studies met all of the inclusion criteria and comprised 24 full-text articles and three conference abstracts [33-35].

Twenty four studies (24) used Pima, two studies used PointCare NOW, one used MyT4 as the index POC CD4 test. Overall, the quality of included studies was considered between moderate and strong. The QUADAS scores ranged from 7 to 12 (of a maximum score of 14) with $63 \%(12 / 19)$ of studies scoring between 10 and 12 . Among 22 studies reporting performance of POC CD4 test (Table 1), 19 (86 \%) were conducted in sub-Saharan countries and three others were in India [36], Brazil [37] and PNG [38]. CD4 predicate testing technologies used as reference include FACSCalibur, FACSCount, Panleucogating (PLG) flow cytometry, Partec CyFlow and GUAVA. Only one study performed duplicate testing [39], and three studies performed precision testing of the reference method on a subset of whole blood samples ( 3 - 15 blood specimens) [40-42]. Among 19 studies reporting diagnostic performance of Pima, only 11 studies provided data required for and then subsequently included in the meta-analyses.

\section{Performance of POC CD4 tests in field settings Failure rates}

For Pima, studies with capillary blood reported a wide range of failure rates from $2 \%$ [43] to $23.3 \%$ [44]. Studies using venous blood in various clinical settings reported failure rates ranging from 4.8 to $15.2 \%$ [40, 44-46]. One study reported a zero "no read" error in laboratory evaluation of Pima with venous blood, however, in field evaluations in different clinical settings failure rate was recorded at a wide range from 6.8 to $20.9 \%$ [22]. For PointCare Now the failure rate varied from $2.9 \%$ in one study [41] to $9.2 \%$ in another [47]. With MyT4 CD4 test a studywide error rate of $9.6 \%$ was recorded [48].

Detailed performance data of Pima is presented in Table 2.

\section{Misclassification, sensitivity and specificity}

When CD4 count testing was conducted using a venous blood specimen Pima showed lower misclassification and higher probabilities of correctly identifying patients eligible for ART across studies and different reference methods. At a CD4 threshold of 350 cells/ $\mu$ l, the total misclassification probability of Pima test using venous blood was 4.0-12.2 \% [44, 49] versus $6.7-17 \%$ for capillary blood $[39,50]$. Pima point estimates for sensitivity and specificity ranged from $89-99 \%$ and $77-93 \%$ for venous blood; and 79-98 \% and 80-99 \% for capillary, respectively. For PointCare Now, one study [47] reported site-specific sensitivity ranging from 38 to $63 \%$, resulting in misclassification of $50 \%$ of patients tested as ineligible for ART; another study [41] reported a lower misclassification of $6 \%$ of patients as ineligible for treatment. For MyT4 CD4, the sensitivity and specificity of the test were 88 and $84 \%$ when compared to FACSCalibur and 95 and $88 \%$ as compared to FACSCount [48]. 
Table 1 Characteristics and Quality assessment of studies included in the review

\begin{tabular}{|c|c|c|c|c|c|c|}
\hline $\begin{array}{l}\text { First author, } \\
\text { year }\end{array}$ & Study population/Study setting & $\begin{array}{l}\text { Study design/ } \\
\text { Sample size }\end{array}$ & Sample & Intervention & Comparison & $\begin{array}{l}\text { Study } \\
\text { quality }^{a}\end{array}$ \\
\hline $\begin{array}{l}\text { Rathunde, } \\
2014 \text { [37] }\end{array}$ & $\begin{array}{l}\text { Adult HIV patients at specialized } \\
\text { ambulatory facilities in academic } \\
\text { tertiary care hospital in Curitiba, } \\
\text { Brazil }\end{array}$ & $\begin{array}{l}\text { Cross-sectional } \\
(N=107)\end{array}$ & Venous & $\begin{array}{l}\text { Alere Pima }{ }^{\mathrm{TM}} \\
\text { CD4 (Pima) }\end{array}$ & FACSCalibur & 9 \\
\hline $\begin{array}{l}\text { Galiwango, } \\
2014 \text { [49] }\end{array}$ & $\begin{array}{l}\text { HIV infected patients (pre and } \\
\text { experienced ART persons) at } \\
\text { field clinics in Rakai, Uganda }\end{array}$ & $\begin{array}{l}\text { Cross-sectional } \\
(N=903)\end{array}$ & Venous & Pima & FACSCalibur & 10 \\
\hline $\begin{array}{l}\text { van Rooyen, } \\
2013[15]\end{array}$ & $\begin{array}{l}\text { Known HIV-positive individuals } \\
\text { older than } 18 \text { years in } \\
\text { KwaZulu-Natal, South Africa (SA) }\end{array}$ & $\begin{array}{l}\text { Prospective } \\
\text { cohort }(N=671)\end{array}$ & $\begin{array}{l}\text { Capillary (Pima); } \\
\text { venous (FACSCalibur) }\end{array}$ & Pima & FACSCalibur & Moderate \\
\hline $\begin{array}{l}\text { Myer, } \\
2013[45]\end{array}$ & $\begin{array}{l}\text { HIV infected pregnant women } \\
\text { at a single large antenatal clinic } \\
\text { in Cape Town, SA }\end{array}$ & $\begin{array}{l}\text { Cross-sectional } \\
(N=546)\end{array}$ & Venous & Pima & $\begin{array}{l}\text { Beckman-Coulter } \\
\text { PLG technology }\end{array}$ & 8 \\
\hline $\begin{array}{l}\text { Mnyani, } \\
2012[51]\end{array}$ & $\begin{array}{l}\text { HIV infected pregnant women } \\
\text { at first ANC visit to Chiawelo } \\
\text { clinic in Johannesburg, SA }\end{array}$ & $\begin{array}{l}\text { Cross-sectional } \\
(N=305)\end{array}$ & $\begin{array}{l}\text { Capillary (Pima); } \\
\text { venous (Ref. tests) }\end{array}$ & Pima & $\begin{array}{l}\text { Beckman Coulter } \\
\text { Flow Cytometer }\end{array}$ & 10 \\
\hline $\begin{array}{l}\text { Mwau, } \\
2013[53]\end{array}$ & $\begin{array}{l}\text { Patients attending } 9 \text { health } \\
\text { facilities (for HIV treatment } \\
\text { and care) offering CD4 count } \\
\text { in Kenya }\end{array}$ & $\begin{array}{l}\text { Cross-sectional } \\
(N=1539)\end{array}$ & $\begin{array}{l}\text { Venous (Pima \& reference } \\
\text { methods); capillary (Pima) }\end{array}$ & Pima & $\begin{array}{l}\text { FACSCount, } \\
\text { Partec Cy-flow, } \\
\text { GUAVA and } \\
\text { FACSCalibur }\end{array}$ & 8 \\
\hline $\begin{array}{l}\text { Glencross, } \\
2012[22]\end{array}$ & $\begin{array}{l}\text { Adult HIV patients attending } \\
\text { (1) Hospital based antenatal } \\
\text { HCT clinic in Johannesburg-phase } \\
\text { II (2) Two Primary health care HCT } \\
\text { clinic in Limpopo province-phase IIIA; } \\
\text { and (3) Inner-city primary health care } \\
\text { clinic in Johannesburg, South } \\
\text { Africa-phase IIIB }\end{array}$ & $\begin{array}{l}\text { Cross-sectional } \\
(N=91 ; N=96 ; \\
N=139)\end{array}$ & Venous and capillary & Pima & $\begin{array}{l}\text { Simplified single } \\
\text { platform (SP) } \\
\text { PLG CD4 }\end{array}$ & 12 \\
\hline $\begin{array}{l}\text { Thakar, } \\
2012[36]\end{array}$ & $\begin{array}{l}\text { HIV positive patients aged } 18-60 \\
\text { attending } 21 \text { ART centers in different } \\
\text { parts of India }\end{array}$ & $\begin{array}{l}\text { Cross-sectional } \\
(N=1790)\end{array}$ & Venous and capillary & Pima & $\begin{array}{l}\text { FACSCalibur; } \\
\text { FACSCount; } \\
\text { Partec CyFlow }\end{array}$ & 12 \\
\hline $\begin{array}{l}\text { Manabe, } \\
2012[46]\end{array}$ & $\begin{array}{l}\text { HIV infected patients at Adult } \\
\text { Infectious Diseases Institute Clinic } \\
\text { in Mulago Hospital, Kampala, Uganda }\end{array}$ & $\begin{array}{l}\text { Cross-sectional } \\
(N=206)\end{array}$ & Venous and capillary & Pima & FACSCalibur & 11 \\
\hline $\begin{array}{l}\text { Jani, } \\
2011[39]\end{array}$ & $\begin{array}{l}\text { HIV infected individuals attending } 2 \\
\text { primary health care ART clinics in } \\
\text { Maputo, Mozambique }\end{array}$ & $\begin{array}{l}\text { Cross-sectional } \\
(N=697)\end{array}$ & $\begin{array}{l}\text { Capillary (Pima); } \\
\text { venous (Ref. tests) }\end{array}$ & Pima & FACSCalibur & 7 \\
\hline $\begin{array}{l}\text { Diaw, } \\
2011[44]\end{array}$ & $\begin{array}{l}\text { Patients (adults \& children; HIV +/-) } \\
\text { presenting for HIV follow-up at three } \\
\text { out-patient clinic \& one lab in Dakar, } \\
\text { Senegal }\end{array}$ & $\begin{array}{l}\text { Cross-sectional } \\
(N=300)\end{array}$ & $\begin{array}{l}\text { Venous ( } 200 \text { patients); } \\
\text { capillary (finger/heel-prick) } \\
100 \text { patients }\end{array}$ & Pima & FACSCount & 11 \\
\hline $\begin{array}{l}\text { Mtapuri- } \\
\text { Zinyowera, } \\
2010[50]\end{array}$ & $\begin{array}{l}\text { Newly diagnosed HIV positive patients } \\
\text { at a VCT center in Harare, Zimbabwe }\end{array}$ & $\begin{array}{l}\text { Cross-sectional } \\
(N=165)\end{array}$ & $\begin{array}{l}\text { Capillary (Pima); } \\
\text { venous (Ref. tests) }\end{array}$ & Pima & FACSCalibur & 11 \\
\hline $\begin{array}{l}\text { Wade, } 2014 \\
\text { [40] }\end{array}$ & $\begin{array}{l}\text { HIV infected patients presenting for } \\
\text { routine CD4 testing at infectious } \\
\text { disease clinic in Dar es Salam, Tanzania }\end{array}$ & $\begin{array}{l}\text { Cross-sectional } \\
(N=200)\end{array}$ & $\begin{array}{l}\text { Capillary (Pima); } \\
\text { venous (Ref. tests) }\end{array}$ & Pima & FACSCalibur & 11 \\
\hline $\begin{array}{l}\text { Wade, } \\
2013 \text { [42] }\end{array}$ & $\begin{array}{l}\text { (Anonymous) HIV infected patients } \\
\text { attending normal CD4 test monitoring } \\
\text { and HIV-negative donors from blood } \\
\text { bank of Regional Hospital and two } \\
\text { Healthcare Centers in Ziguinchor, } \\
\text { Senegal }\end{array}$ & $\begin{array}{l}\text { Cross-sectional } \\
(N=128)\end{array}$ & Venous & Pima & FACSCount & 11 \\
\hline Gous, & HIV infected patients $>18$ years old & Cross-sectional & Capillary (Pima); & Pima & PLG CD4 FC 500 & 10 \\
\hline
\end{tabular}


Table 1 Characteristics and Quality assessment of studies included in the review (Continued)

\begin{tabular}{|c|c|c|c|c|c|c|}
\hline $\begin{array}{l}\text { Malagun, } \\
2014 \text { [38] }\end{array}$ & $\begin{array}{l}\text { HIV infected adults }>18 \text { year old } \\
\text { attending one urban (Heduru HIV } \\
\text { clinic at Port Moresby General Hospital, } \\
\text { Port Moresby) and } 2 \text { rural } \\
\text { (Asaro District Health Centre and } \\
\text { Kainantu Rural Hospital) clinics in } \\
\text { Papua New Guinea }\end{array}$ & $\begin{array}{l}\text { Cross-sectional } \\
(N=237)\end{array}$ & Venous & Pima & FACSCount & 11 \\
\hline $\begin{array}{l}\text { Picken, } \\
2014 \text { [43] }\end{array}$ & $\begin{array}{l}\text { HIV positive mother of children } \\
\text { with CD } 4 \text { count }<500 \text { cells/ } \mu \text { land } \\
\text { not on ART at Tygerberg hospital, } \\
\text { Cape Town, SA }\end{array}$ & $\begin{array}{l}\text { Cross-sectional } \\
(N=52)\end{array}$ & $\begin{array}{l}\text { Capillary (Pima); } \\
\text { venous (Ref. tests) }\end{array}$ & Pima & $\begin{array}{l}\text { Beckman Coulter } \\
\text { FC } 500 \mathrm{MPL}^{\oplus}\end{array}$ & 11 \\
\hline $\begin{array}{l}\text { Mwau, } \\
2014[48]\end{array}$ & $\begin{array}{l}\text { HIV infected patients } \geq 18 \text { years old } \\
\text { at Comprehensive clinics of } 2 \text { health } \\
\text { care facilities in Busia county of } \\
\text { Western province, Kenya }\end{array}$ & $\begin{array}{l}\text { Cross-sectional } \\
(\mathrm{N}=276)\end{array}$ & $\begin{array}{l}\text { Capillary (Pima); } \\
\text { venous (Ref. tests) }\end{array}$ & $\begin{array}{l}\text { MyT4 POC } \\
\text { CD4 }\end{array}$ & $\begin{array}{l}\text { FACSCalibur and } \\
\text { FACSCount }\end{array}$ & 11 \\
\hline $\begin{array}{l}\text { Gumbo, } \\
2013 \text { [41] }\end{array}$ & $\begin{array}{l}\text { HIV infected adult patients attending } \\
\text { Harare Central hospital opportunistic } \\
\text { infection clinic, Zimbabwe }\end{array}$ & $\begin{array}{l}\text { Cross-sectional } \\
(N=104)\end{array}$ & Venous & $\begin{array}{l}\text { PointCare } \\
\text { NOW }\end{array}$ & BD FACSCalibur & 9 \\
\hline $\begin{array}{l}\text { Bergeron, } \\
2012[47]\end{array}$ & $\begin{array}{l}\text { HIV infected adult patients; unknown } \\
\text { HIV status; and HIV infected children } \\
\text { aged 12-59 months attending: } \\
\text { (1) the Instituto Nacional de Saude } \\
\text { (INS) Maputo, Mozambique; (2) the } \\
\text { Institute of Tropical Medicine of } \\
\text { Antwerp (Belgium) in Tete, } \\
\text { Mozambique; (3) Wits University, } \\
\text { Johannesburg, SA }\end{array}$ & $\begin{array}{l}\text { Cross-sectional } \\
(N=472)\end{array}$ & Venous & $\begin{array}{l}\text { PointCare } \\
\text { NOW }\end{array}$ & $\begin{array}{l}\text { FACSCalibur and } \\
\text { the EPICS- XL }\end{array}$ & 8 \\
\hline $\begin{array}{l}\text { Zeh, } \\
2014 \text { [35] }\end{array}$ & HIV infected patients in Western Kenya & $\begin{array}{l}\text { Cross-sectional } \\
(N=147)\end{array}$ & Venous and capillary & Pima & FACSCalibur & - \\
\hline $\begin{array}{l}\text { Arnet N, } \\
2013[33]\end{array}$ & $\begin{array}{l}\text { HIV infected patients from } 5 \text { PMTCT } \\
\text { and HIV treatment sites in } \\
\text { Dar-es-Salaam, Tanzania }\end{array}$ & $\begin{array}{l}\text { Cross-sectional } \\
(N=1060)\end{array}$ & Venous and capillary & Pima & FACSCalibur & - \\
\hline
\end{tabular}

HCT HIV counseling and testing, ART Antiretroviral therapy, VCT Voluntary counseling and testing; PMTCT Prevention of mother to child transmission ${ }^{a}$ Study quality assessment using EPHPP tool (strong/moderate/weak), or QUADAS score (out of 14)

Meta-analysis of diagnostic accuracy of the Alere Pima $^{\mathrm{TM}}$ CD4 in field testing We aimed to conduct categorical data analysis of diagnostic accuracy of Pima at CD4 350 and 500 cells/ $\mu$ l cut-offs. However, only three included studies [40, 42, 49] reported data required for meta-analysis at the 500 cut-off. Thus only analysis at a CD4 threshold of 350 cells/ $\mu$ l was performed. Required data including number of true positive, false positive, false negative and true negative cases were reported in the literature from 9 studies [38, 40, 42, 43, 45, 49-52]. Data from two studies $[37,53]$ were received from the authors following email contact, yielding a final dataset comprising 11 studies for the meta-analysis. Among these, two studies $[40,53]$ reported Pima test results for both venous and capillary blood, and these data were treated in meta-analyses as independent study results but with model standard errors corrected for the lack of independence in observations. Five studies [37, 38, 42, 45,49 ] reported the results with venous and four studies [43, 50-52] with capillary blood only.

Examination of post-estimation of diagnostic statistics after preliminary meta-analyses provided some evidence of model outlier bias, with two studies [53] (capillary) and [38] (venous) showing model discrepant test sensitivity and specificity (Cook's distances: 2.12 and 3.65 respectively). However, diagnostic test data from these studies were included in the pooled meta-analysis after sensitivity analysis, with outlying cases excluded, indicated no marked difference in pooled estimates (included versus excluded sensitivity and specificity: 92 vs. $92 \%$ and 87 vs. $88 \%$, respectively).

Diagnostic accuracy of the test in field settings was relatively high, with pooled sensitivity and specificity estimated at $92 \%(95 \% \mathrm{CI}=88-95 \%)$ and $87 \%(95 \%$ $\mathrm{CI}=85-88 \%$ ) respectively (Fig. 2). Further, pooled positive and negative likelihood ratios were also at levels indicating relatively strong diagnostic performance of Pima $(+\mathrm{LR}=7.0,95 \% \mathrm{CI}=6.1-7.9 ;-\mathrm{LR}=$ 0.09, $95 \% \mathrm{CI}=0.06-0.13)$. Figure 3 shows observed sensitivity and specificity plotted for each included study with the HSROC curve, the pooled estimate and $95 \%$ confidence and prediction contours.

Bivariate random-effect hierarchical models estimating pooled diagnostic performance with a covariate for blood sample type showed some potential difference in summary sensitivity and specificity by blood sample 
Table 2 Performance of Pima stratified by venous and capillary blood collection and presented by reference test used

\begin{tabular}{|c|c|c|c|c|c|c|c|c|c|}
\hline \multirow{3}{*}{$\begin{array}{l}\text { Author, } \\
\text { year }\end{array}$} & \multicolumn{4}{|c|}{ Performance of Pima on capillary blood } & \multirow[t]{3}{*}{ Author, year } & \multicolumn{4}{|c|}{ Performance of Pima on venous blood } \\
\hline & \multirow[t]{2}{*}{ Bias/LoA; Sample size (N) } & \multirow{2}{*}{$\begin{array}{l}\text { Sensitivity } \\
\text { Specificity }\end{array}$} & \multirow{2}{*}{$\begin{array}{l}\text { Total } \\
\text { Misclassification }\end{array}$} & \multirow{2}{*}{$\begin{array}{l}\text { Failure } \\
\text { rate }\end{array}$} & & \multirow[t]{2}{*}{ Bias/LoA Sample size (N) } & Sensitivity & \multirow{2}{*}{$\begin{array}{l}\text { Total } \\
\text { Misclassification }\end{array}$} & \multirow[t]{2}{*}{ Failure rate } \\
\hline & & & & & & & Specificity & & \\
\hline \multicolumn{10}{|c|}{ Reference test $=$ FACSCalibur } \\
\hline $\begin{array}{l}\text { (van } \\
\text { Rooyen, } \\
\text { Barnabas } \\
\text { et al. 2013) } \\
{[14]}\end{array}$ & 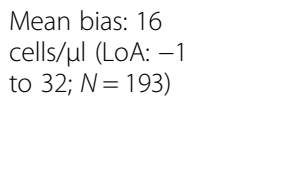 & $\begin{array}{l}\text { Not reported } \\
\text { (NR) }\end{array}$ & $N R$ & $N R$ & $\begin{array}{l}\text { (Rathunde, } \\
\text { Kussen et al. } \\
\text { 2014) [32] }\end{array}$ & Bias/LoA NR; N= 107 & $\begin{array}{l}\text { At CD4 threshold } \\
\text { of } 350 \text { cells/ } / \text { l: } \\
\text { Sensitivity } 94 \% \\
\text { Specificity } 93 \%\end{array}$ & $N R$ & $N R$ \\
\hline \multirow{5}{*}{$\begin{array}{l}\text { (Jani, Sitoe } \\
\text { et al. 2011) } \\
\text { [34] }\end{array}$} & $\begin{array}{l}\text { Accurate absolute } \\
\text { counts }\end{array}$ & \multirow[t]{5}{*}{$N R$} & \multirow{2}{*}{ 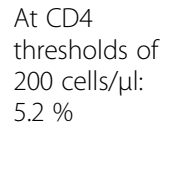 } & \multirow[t]{5}{*}{$N R$} & \multirow{5}{*}{$\begin{array}{l}\text { (Galiwango, } \\
\text { Lubyayi et } \\
\text { al. 2014) } \\
{[45]}\end{array}$} & \multirow{2}{*}{$\begin{array}{l}\text { Pima significantly } \\
\text { underestimate CD4 } \\
\text { count particularly } \\
\text { at higher CD4 count. }\end{array}$} & \multirow{2}{*}{ 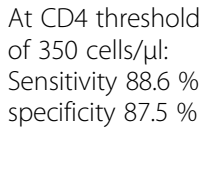 } & \multirow[t]{2}{*}{$12.2 \%$} & \multirow[t]{5}{*}{ NR } \\
\hline & $\begin{array}{l}\text { Bias: }-52.8 \text { cell/ } \mu \mathrm{l} \\
\text { (LoA: }-250.9 \\
\text { to } 145.2 ; N=135) \text {. }\end{array}$ & & & & & & & & \\
\hline & \multirow{3}{*}{$\begin{array}{l}\text { Bias was smaller } \\
\text { at lower CD4 } \\
\text { count (<500: }-24.4) \\
\text { than at higher CD4 } \\
\text { count ( }>500:-107.9) \text {. }\end{array}$} & & \multirow{3}{*}{$\begin{array}{l}\text { At CD4 } \\
\text { thresholds of } \\
350 \text { cells/ } / \text { l: } \\
17 \%\end{array}$} & & & $\begin{array}{l}\text { Bias: }-34.6 \text { cells } / \mu l \\
(\text { LoA: }-219.8 \text { to }+150.6 ; \\
N=903)\end{array}$ & \multirow{3}{*}{ 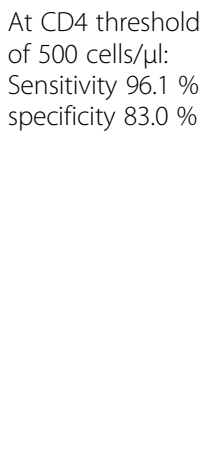 } & \multirow[t]{3}{*}{$9.5 \%$} & \\
\hline & & & & & & 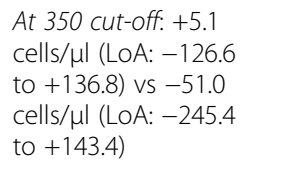 & & & \\
\hline & & & & & & 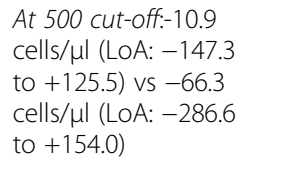 & & & \\
\hline \multirow[t]{3}{*}{$\begin{array}{l}\text { (Mtapuri- } \\
\text { Zinyowera, } \\
\text { Chideme } \\
\text { et al. 2010) } \\
\text { [46] }\end{array}$} & $\begin{array}{l}\text { Mean bias: } 7.6 \\
\text { cells/ } / \mu \text { l } \\
(\text { LoA: }-173.8 \\
\text { to }+189.0)\end{array}$ & $\begin{array}{l}\text { At CD4 threshold } \\
\text { of } 200 \text { cells/ } \mu \text { : } \\
\text { Sensitivity: } 95.1 \% \\
\text { Specificity: } 91.6 \%\end{array}$ & $6.7 \%$ & $N R$ & $\begin{array}{l}\text { (Mwau, } \\
\text { Adungo et } \\
\text { al. 2013) } \\
\text { [49] }\end{array}$ & $\begin{array}{l}\text { Bias: }-64.8 \text { cells/ } / \mu l \\
\text { (LoA: }-332.5 \text { to }+203.0 ; \\
N=396)\end{array}$ & $\begin{array}{l}\text { At CD4 threshold } \\
\text { of } 350 \text { cells/ } / \mu \text { (in } \\
\text { those } \geq 5 \text { years } \\
\text { old } N=389 \text { ): } \\
\text { Sensitivity: } 89.7 \% \\
\text { Specificity: } 87 \%\end{array}$ & $11.9 \%(47 / 396)$ & $N R$ \\
\hline & \multirow[t]{2}{*}{ 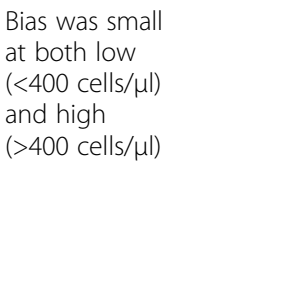 } & $\begin{array}{l}\text { With sub- } \\
\text { samples of FACS- } \\
\text { Calibur results of } \\
(100 \text { to } 300 \text { cells/ } \\
\mu l)\end{array}$ & $12.8 \%$ & & & & \multirow[t]{2}{*}{$\begin{array}{l}\text { At CD4 threshold } \\
\text { of } 200 \text { cells/ } / \mu \text { l } \\
\text { (in those } \geq 5 \text { years } \\
\text { old } N=389 \text { ): } \\
\text { Sensitivity: } 86.7 \% \\
\text { Specificity: } 94.1 \%\end{array}$} & \multirow[t]{2}{*}{$N R$} & \\
\hline & & $\begin{array}{l}\text { At CD4 threshold } \\
\text { of } 350 \text { cells/ } \mu \text { l: } \\
\text { Sensitivity: } 94.7 \% \\
\text { Specificity: } 87.5 \%\end{array}$ & $6.7 \%$ & & & & & & \\
\hline
\end{tabular}


Table 2 Performance of Pima stratified by venous and capillary blood collection and presented by reference test used (Continued)

\begin{tabular}{|c|c|c|c|c|c|c|c|c|c|}
\hline & & $\begin{array}{l}\text { With sub- } \\
\text { samples of FACS- } \\
\text { Calibur results of } \\
\text { (200 to } 500 \text { cells/ } \\
\mu \text { l) }\end{array}$ & $14.1 \%$ & & & & & & \\
\hline $\begin{array}{l}\text { (Thakar, } \\
\text { Mahajan et } \\
\text { al. 2012) } \\
\text { [31] }\end{array}$ & $\begin{array}{l}\text { Relative bias: }-9.1 \% \\
\text { LoA: }-46 \% \text { to } 27 \% \\
N=175\end{array}$ & $N R$ & $N R$ & $N R$ & $\begin{array}{l}\text { (Thakar, } \\
\text { Mahajan et } \\
\text { al. 2012) } \\
{[31]}\end{array}$ & $\begin{array}{l}\text { Among patients with } \\
\text { CD4<350 cells/ } \mu \text { l: } \\
\text { relative bias: }+4 \% \\
(N=121)\end{array}$ & 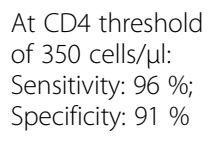 & $N R$ & $N R$ \\
\hline \multirow[t]{2}{*}{$\begin{array}{l}\text { (Manabe, } \\
\text { Wang et al. } \\
\text { 2012) [41] }\end{array}$} & $\begin{array}{l}\text { Bias: }-66.3 \text { cells/ } \mu l \\
(\text { LoA: }-83.4 \text { to }+49.2 \\
p<0.001 ; N=176)\end{array}$ & $N R$ & $N R$ & $17.7 \%$ & $\begin{array}{l}\text { (Manabe, } \\
\text { Wang et al. } \\
\text { 2012) [41] }\end{array}$ & 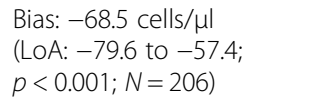 & $N R$ & $N R$ & $8.1 \%$ \\
\hline & 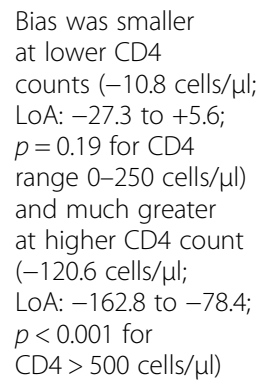 & & & & & 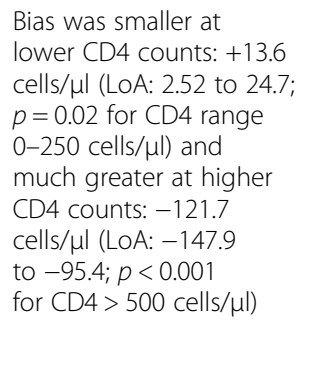 & & & \\
\hline \multirow[t]{3}{*}{$\begin{array}{l}\text { (Wade, } \\
\text { Daneau et } \\
\text { al. 2014) } \\
{[35]}\end{array}$} & $\begin{array}{l}\text { Relative bias: }-0.9 \% ; \\
(\text { LoA: }-57.3 \text { to }+55.6) ; \\
N=200\end{array}$ & 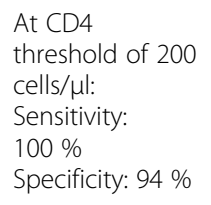 & $4 \%(16 / 410)$ & $\begin{array}{l}4.5 \%(9 / \\
200)\end{array}$ & $\begin{array}{l}\text { (Wade, } \\
\text { Daneau et } \\
\text { al. 2014) } \\
{[35]}\end{array}$ & $\begin{array}{l}\text { Relative mean bias: }-9.4 \\
\% \text { (LoA: }-54.4 \text { to }+35.6)\end{array}$ & $\begin{array}{l}\text { At CD4 threshold } \\
\text { of } 200 \text { cells/ml: } \\
\text { Sensitivity: } 98 \% \\
\text { Specificity: } 95 \%\end{array}$ & $3 \%(14 / 440)$ & $6.5 \%(13 / 200)$ \\
\hline & \multirow{2}{*}{$\begin{array}{l}\text { Sub-samples of } \\
\text { CD4 } \leq 200: 5 \% \\
(-78 \text { to }+89) ; \\
\text { CD4 200-500: } 0 \% \\
(-49 \text { to +49); } \\
\text { CD4 } \geq 500:-8 \% \\
(-49 \text { to }+34)\end{array}$} & 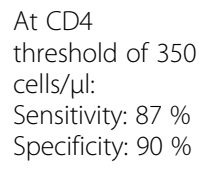 & $13.4 \%(55 / 410)$ & & & \multirow{2}{*}{$\begin{array}{l}\text { Sub-samples of CD4 } \leq 200: \\
1 \% \text { (LoA: }-75 \text { to } 77) ; \\
\text { CD4 200-500: }-11 \% \\
\text { (LoA: }-46 \text { to }+25) ; \\
\text { CD4 } \geq 500:-15 \% \\
\text { (LoA: }-34 \text { to }+4)\end{array}$} & $\begin{array}{l}\text { At CD4 threshold } \\
\text { of } 350 \text { cells/Ml: } \\
\text { Sensitivity: } 97 \% \\
\text { Specificity: } 80 \%\end{array}$ & $9 \%(40 / 440)$ & \\
\hline & & 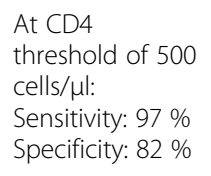 & $N R$ & & & & 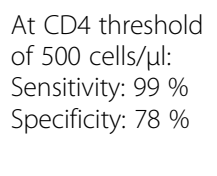 & NR & \\
\hline $\begin{array}{l}\text { (Zeh, } \\
\text { Inzaule et } \\
\text { al. 2014) } \\
\text { [30] }\end{array}$ & $\begin{array}{l}\text { Bias: }-44 \text { cells } / \mu l \\
N=147\end{array}$ & $\begin{array}{l}\text { At CD4 } \\
\text { threshold of } 350 \\
\text { cells/ } \mu \text { l: } \\
\text { Sensitivity: } 86 \% \\
\text { Specificity: } 99 \%\end{array}$ & $N R$ & $N R$ & $\begin{array}{l}\text { (Zeh, } \\
\text { Inzaule et } \\
\text { al. 2014) } \\
{[30]}\end{array}$ & Bias: -86 cells/ul; $N=147$ & $\begin{array}{l}\text { At CD4 threshold } \\
\text { of } 350 \text { cells/ml: } \\
\text { Sensitivity: } 94 \% \\
\text { Specificity: } 95 \%\end{array}$ & $N R$ & $N R$ \\
\hline
\end{tabular}


Table 2 Performance of Pima stratified by venous and capillary blood collection and presented by reference test used (Continued)

\begin{tabular}{|c|c|c|c|c|c|c|c|c|c|}
\hline $\begin{array}{l}\text { (Arnett N } \\
\text { 2013) [28] }\end{array}$ & 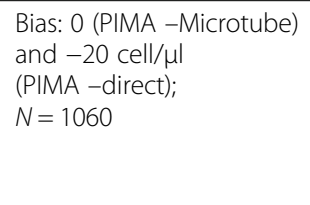 & $N R$ & $N R$ & $\begin{array}{l}8.6 \% \\
\text { (Micro- } \\
\text { tube) } \\
\text { and } \\
10.1 \% \\
\text { (direct) }\end{array}$ & $\begin{array}{l}\text { (Arnett N } \\
\text { 2013) [28] }\end{array}$ & Bias: -10 cells $/ \mu \mathrm{l}$ & $N R$ & $N R$ & $7.7 \%$ \\
\hline \multicolumn{10}{|c|}{ Reference test $=$ FACSCount } \\
\hline \multirow[t]{3}{*}{$\begin{array}{l}\text { (Mwau, } \\
\text { Adungo et } \\
\text { al. 2013) } \\
\text { [49] }\end{array}$} & \multirow[t]{3}{*}{ 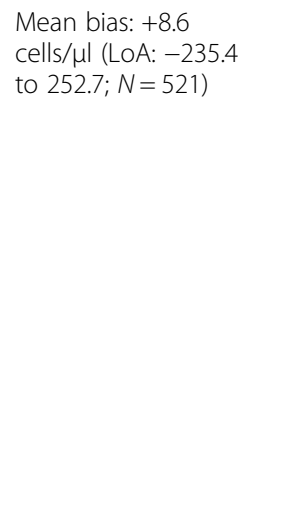 } & \multirow[t]{3}{*}{$\begin{array}{l}\text { At CD4 } \\
\text { threshold of } 350 \\
\text { cells/ } \mu \text { l: } \\
\text { Sensitivity: } \\
79.4 \% \\
\text { Specificity: } \\
86.9 \%\end{array}$} & \multirow[t]{3}{*}{$16.5 \%(86 / 521)$} & \multirow[t]{3}{*}{$N R$} & $\begin{array}{l}\text { (Mwau, } \\
\text { Adungo et } \\
\text { al. 2013) } \\
{[49]}\end{array}$ & \multirow[t]{2}{*}{$\begin{array}{l}\text { Mean bias: }+7.8 \\
\text { cells/ } / \mu \text { l }(\text { LoA: }-168.9 \\
\text { to } 184.4 ; N=822)\end{array}$} & $\begin{array}{l}\text { At CD4 threshold } \\
\text { of } 350 \text { cells/ } / \mu \text { l } \\
\text { (in those } \geq 5 \text { years } \\
\text { old } N=813 \text { ): } \\
\text { Sensitivity: } 79.4 \% \\
\text { Specificity: } 83.4 \%\end{array}$ & $N R$ & \multirow[t]{3}{*}{$N R$} \\
\hline & & & & & & & $\begin{array}{l}\text { At CD4 of } 200 \\
\text { cells/ } / \mu \text { l (in those } \geq \\
5 \text { years old } N=813 \text { ): } \\
\text { Sensitivity: } 83 \% \\
\text { Specificity: } 98.2 \%\end{array}$ & $N R$ & \\
\hline & & & & & $\begin{array}{l}\text { (Thakar, } \\
\text { Mahajan et } \\
\text { al. 2012) } \\
{[31]}\end{array}$ & $\begin{array}{l}\text { Among patients with } \\
\text { CD4<350 cells/ } \mu \text { : } \\
\text { Mean relative bias: }-5 \% \\
(N=206)\end{array}$ & $\begin{array}{l}\text { At CD4 threshold } \\
\text { of } 350 \text { cells/ml: } \\
\text { Sensitivity: } 92 \% \text {; } \\
\text { Specificity: } 91 \%\end{array}$ & $N R$ & \\
\hline \multirow[t]{9}{*}{$\begin{array}{l}\text { (Diaw, } \\
\text { Daneau et } \\
\text { al. 2011) } \\
\text { [39] }\end{array}$} & \multirow[t]{2}{*}{$\begin{array}{l}\text { Of } 95 \text { HIV }(+) \text { patients, } \\
\text { Absolute bias: }-39 \\
\text { cells/ } \mu \text { l (LoA: }-258 \\
\text { to }+179)\end{array}$} & 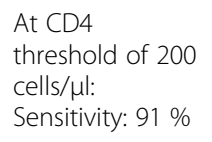 & \multirow[t]{2}{*}{$\begin{array}{l}5.3 \%(5 / 95) ; \text { of } \\
\text { finger-prick } \\
\text { samples }\end{array}$} & \multirow{9}{*}{$\begin{array}{l}14 \% \\
\text { total; } \\
23 \% \text { in } \\
\text { one } \\
\text { study } \\
\text { site }\end{array}$} & \multirow[t]{6}{*}{$\begin{array}{l}\text { (Diaw, } \\
\text { Daneau et } \\
\text { al. 2011) } \\
{[39]}\end{array}$} & \multirow[t]{2}{*}{$\begin{array}{l}\text { For } 100 \mathrm{HIV}(+) \text { patients, } \\
\text { Absolute bias: }-32 \\
\text { cells/Ml (LoA: }-146 \\
\text { to }+84)\end{array}$} & 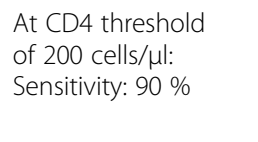 & \multirow[t]{2}{*}{$4 \%$} & \multirow[t]{6}{*}{$4.8 \%$} \\
\hline & & Specificity: 97 \% & & & & & Specificity: 98 \% & & \\
\hline & \multirow{7}{*}{ 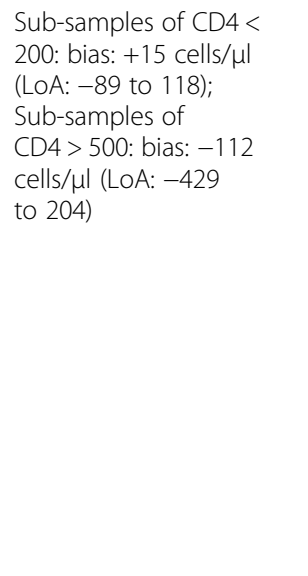 } & \multirow{7}{*}{ 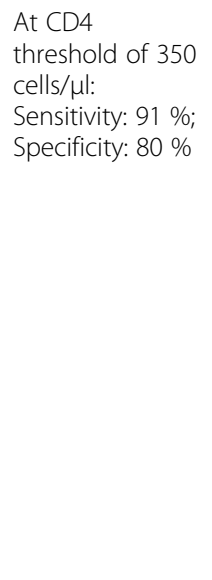 } & \multirow{7}{*}{ NR } & & & \multirow{3}{*}{ 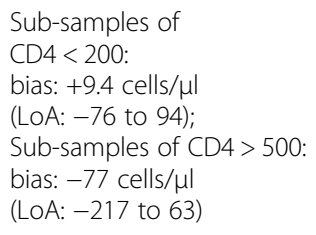 } & $\begin{array}{l}\text { At CD4 threshold } \\
\text { of } 350 \text { cells/ } / \mu l:\end{array}$ & \multirow[t]{4}{*}{$N R$} & \\
\hline & & & & & & & Sensitivity: $98 \%$ & & \\
\hline & & & & & & & Specificity: $79 \%$ & & \\
\hline & & & & & & $\begin{array}{l}\text { For } 99 \text { HIV }(-) \text { controls } \\
\text { Absolute bias: }-125 \\
\text { cells/ } / \mu \text { l (LoA: }-434 \\
\text { to }+184 \text { cells } / \mu \text { for } \\
\text { all ranges of CD4 }\end{array}$ & & & \\
\hline & & & & & \multirow{3}{*}{$\begin{array}{l}\text { (Wade, } \\
\text { Diaw et al. } \\
\text { 2013) [37] }\end{array}$} & \multirow{3}{*}{$\begin{array}{l}\text { Bias: }-30 \text { cells } / \mu l \\
\text { (LoA: }-160 \text { to } 101 ; \\
N=128: 111 \mathrm{HIV}+\& \\
17 \text { HIV-) }\end{array}$} & $\begin{array}{l}\text { At CD4 threshold } \\
\text { of } 200 \text { cells/ } / \text { l: }\end{array}$ & \multirow[t]{3}{*}{$N R$} & \multirow[t]{3}{*}{ NR } \\
\hline & & & & & & & Sensitivity $95 \%$ & & \\
\hline & & & & & & & Specificity $96 \%$ & & \\
\hline
\end{tabular}


Table 2 Performance of Pima stratified by venous and capillary blood collection and presented by reference test used (Continued)

Reference test $=$ Beckman-Coulter flow cytometry using Pan-leucogating (PLG) method

\begin{tabular}{|c|c|}
\hline \multirow[t]{2}{*}{$\begin{array}{l}\text { (Mnyani, } \\
\text { Mclntyre et } \\
\text { al. 2012) } \\
{[47]}\end{array}$} & $\begin{array}{l}\text { Bias: }-20.5 \text { cells/ } / \mathrm{l} \\
(\text { LoA: }-175.0 \\
\text { to }+133.9 ; \\
p<0.001 ; \\
N=296)\end{array}$ \\
\hline & $\begin{array}{l}\text { No significant } \\
\text { variability in the } \\
\text { level of agreement } \\
\text { related to age and } \\
\text { gestational age }\end{array}$ \\
\hline \multirow[t]{2}{*}{$\begin{array}{l}\text { (Glencross, } \\
\text { Coetzee et } \\
\text { al. 2012) } \\
{[42]}\end{array}$} & $\begin{array}{l}\text { Phase II (Hospital } \\
\text { ANC clinic: Bias: }-37.9 \\
\text { cells/ } \mu \text { l (LoA: }-389.1 \\
\text { to } 309.8 ; N=77\end{array}$ \\
\hline & $\begin{array}{l}\text { Phase IIIA Rural/poor } \\
\text { resourced clinic: } \\
\text { Not applicable (NA) }\end{array}$ \\
\hline
\end{tabular}

$10.8 \%$; mostly NR

At CD4

threshold of 350 in favor of

cells/ $\mu$ :

patient

treatment.

Sensitivity: $93 \%$

(95\% Cl 87-96),

Specificity: $86 \%$

(95\% Cl 80-91)

NR

NA

Not applicable (NA)
Sub-samples of CD4 < 200: At CD4 threshold

Bias: +6.0 cells $/ \mu \mathrm{l}$

of 350 cells/ul:

(LoA: -39 to +51 )

Sensitivity $97 \%$

Specificity $90 \%$

Sub-samples of

CD4 > 500: Bias: -65

At CD4 threshold

of 500 cells $/ \mu$.:

Sensitivity $99 \%$

Specificity $72 \%$

(Malagun, Urban clinic: Bias: -46.4 At CD4 threshold

to 107.0$) ; N=139$

of 350 cells $/ \mu$ :

$10.7 \%$

Error rate:

Sensitivity: $99.2 \%$;

$5.1 \%$

Rural clinic: Bias: -55.8

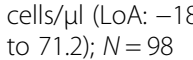

specificity: $77.1 \%$

(Myer, Bias: -22.7 cells/ $\mu$ At CD4 threshold

Daskilewicz (LoA: -174.6 to 129.2); $\quad$ of 350 cells/ $/$ l:

$10 \%$

$61 / 546$ samples required 83

additional test;

4 returned no

result due to

repeated

Bias increased with $\quad$ Sensitivity: $92 \%$

increasing gestational

age

Specificity: $89 \%$;

machine errors

\section{Sensitivity \& specificity}

did not vary significantly

across gestational age

$\begin{array}{ll}\text { (Glencross, } & \text { Phase II (Hospital ANC } \\ \text { Coetzee et } & \text { clinic: Bias: }-19.6 \text { cells/ } \mu \text { l }\end{array}$

$N R$

$10.4 \%(5 / 48) \&$

$20.9 \%(9 / 43)$

for 2 devices

$\begin{array}{ll}\text { [42] } & N=91)\end{array}$

Substantial, clinically

significant difference

to predicate: Bia

cells/ $\mu$ l (LoA -336.1

to $547.5 ; N=96$ )

Larger bias and wider

LoA for samples with

CD4 < 350: + 131.4

cells/Ml (LoA: -275.8

to $+538.6 ; N=32$ ) as

compared to samples

with CD4 < 500: +102.3

cells/ $\mu$ l (LoA: -289 to

493.6; $N=52)=>$ increasing 
Table 2 Performance of Pima stratified by venous and capillary blood collection and presented by reference test used (Continued)

\begin{tabular}{|c|c|c|c|c|c|c|c|c|c|}
\hline & & & & & & \multicolumn{4}{|l|}{$\begin{array}{l}\text { error at CD4 range of } \\
\text { less than } 350 \text { cells/ } \mu \mathrm{l}\end{array}$} \\
\hline & $\begin{array}{l}\text { Phase IIIB well resourced } \\
\text { clinic: NA }\end{array}$ & $N A$ & $N A$ & NA & & \multicolumn{3}{|l|}{ 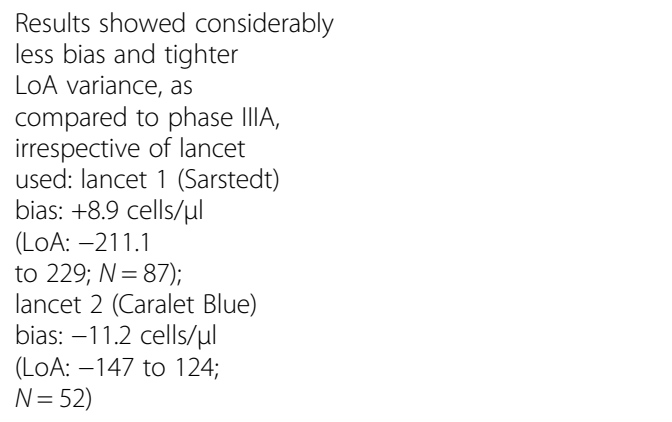 } & $9 \%(14 / 153)$ \\
\hline \multirow[t]{2}{*}{$\begin{array}{l}\text { (Gous, } \\
\text { Scott et al. } \\
\text { 2013) [48] }\end{array}$} & 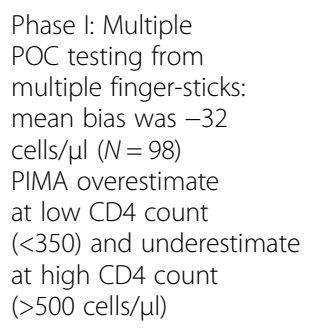 & $\begin{array}{l}\text { At CD4 } \\
\text { threshold of } 350 \\
\text { cells/ul: } \\
\text { Sensitivity } \\
86.4 \%, \\
\text { Specificity } \\
88.5 \%\end{array}$ & $12.4 \%$ & \multicolumn{2}{|l|}{$16.3 \%$} & NA & NA & NA & NA \\
\hline & $\begin{array}{l}\text { Phase II: Multiple } \\
\text { POC testing from } \\
\text { single finger-stick: } \\
\text { Mean bias - 30 } \\
\text { cells/ } / \mu l(N=73)\end{array}$ & $\begin{array}{l}\text { At CD4 } \\
\text { threshold of } 350 \\
\text { cells/ } / \text { l: } \\
\text { Sensitivity } \\
97.5 \% \text {, } \\
\text { Specificity } 95 \%\end{array}$ & $4.1 \%$ & \multicolumn{2}{|l|}{$19.2 \%$} & NA & NA & NA & NA \\
\hline $\begin{array}{l}\text { (Picken, } \\
\text { Williams et } \\
\text { al. 2014) } \\
{[38]}\end{array}$ & $\begin{array}{l}\text { Bias: } 23.8 \text { cells } / \mu l \\
\text { (LoA: }-166.1 \text { to } \\
213.8 ; N=50\end{array}$ & 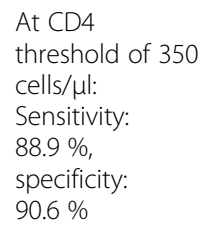 & $10 \%$ & \multicolumn{4}{|l|}{$1.9 \%$} & & \\
\hline \multicolumn{10}{|c|}{ Reference test $=$ Partec Cyflow } \\
\hline $\begin{array}{l}\text { (Mwau, } \\
\text { Adungo et } \\
\text { al. 2013) } \\
{[49]}\end{array}$ & $\begin{array}{l}\text { Mean bias: }-10.0 \\
\text { cells/ } \mu \text { (LoA: }-261.4 \\
\text { to } 241.4 ; N=162)\end{array}$ & $N R$ & $N R$ & $N R$ & $\begin{array}{l}\text { (Mwau, } \\
\text { Adungo et } \\
\text { al. 2013) } \\
\text { [49] }\end{array}$ & $\begin{array}{l}\text { Mean bias: }-24.2 \text { cells } / \mu l \\
(\text { LoA: }-277.6 \text { to }+229.3 ; N \\
=407)\end{array}$ & NR & $N R$ & $N R$ \\
\hline
\end{tabular}


Table 2 Performance of Pima stratified by venous and capillary blood collection and presented by reference test used (Continued)

\begin{tabular}{|c|c|c|c|c|c|c|c|c|c|}
\hline & & & & & $\begin{array}{l}\text { (Thakar, } \\
\text { Mahajan et } \\
\text { al. 2012) } \\
\text { [31] }\end{array}$ & $\begin{array}{l}\text { Among patients with CD4 } \\
<350 \text { cells/ } \mu \text { l: mean } \\
\text { relative bias }+8 \%(N= \\
550)\end{array}$ & $\begin{array}{l}\text { At CD4 } 350 \\
\text { threshold: } \\
\text { Sensitivity: } 91 \% ; \\
\text { Specificity: } 96 \%\end{array}$ & $N R$ & $N R$ \\
\hline \multicolumn{10}{|c|}{ Reference test = GUAVA } \\
\hline $\begin{array}{l}\text { (Mwau, } \\
\text { Adungo et } \\
\text { al. 2013) } \\
\text { [49] }\end{array}$ & $\begin{array}{l}\text { Mean bias: }+23.9 \\
\text { cells/ } \mu \text { l }(\text { LOA }-329.6 \\
\text { to } 281.9 ; N=176)\end{array}$ & $N R$ & $N R$ & $N R$ & $\begin{array}{l}\text { (Mwau, } \\
\text { Adungo et } \\
\text { al. 2013) } \\
{[49]}\end{array}$ & $\begin{array}{l}\text { Mean bias: }-0.3 \text { cells } / \mu l \\
\text { (LoA: }-315.0 \text { to } 315.6 ; N= \\
\text { 191) }\end{array}$ & $N R$ & $N R$ & $N R$ \\
\hline
\end{tabular}




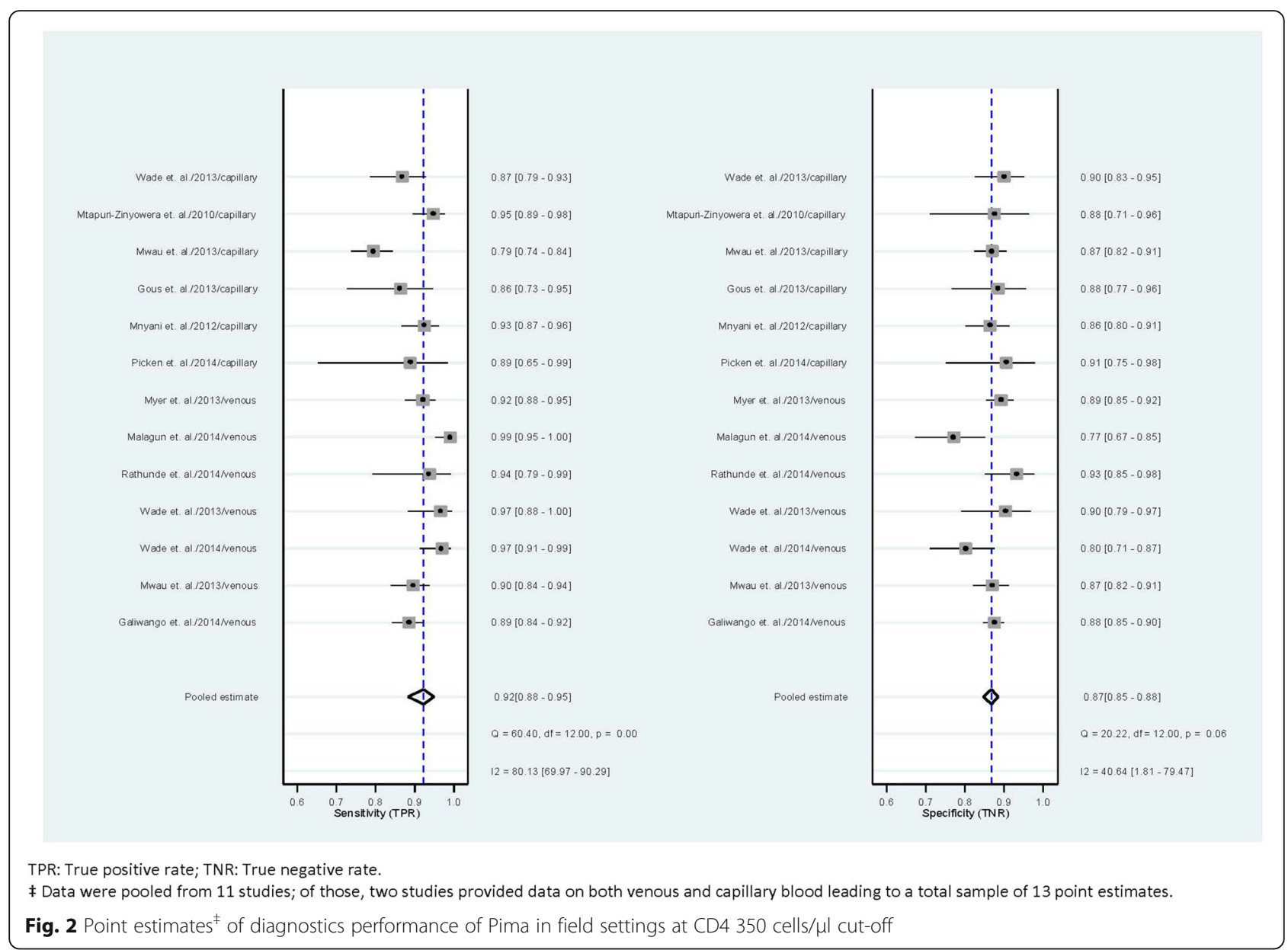

type. Using venous samples, pooled sensitivity was $94 \%$ (95\% CI = 89-97\%) and pooled specificity $86 \%$ (95\% $\mathrm{CI}=82-89 \%)$, while using capillary blood pooled sensitivity was $89 \%(95 \% \mathrm{CI}=83-93 \%)$ and specificity $87 \%$ (95\% CI $=86-89 \%$ ). However, a post-estimation test of the joint effect of blood sample type on the sensitivity and specificity of Pima showed that these differences in diagnostic accuracy did not reach statistical significance (Wald $\chi^{2}(2)=4.77, p=0.09$ ).

\section{Bias and limit of agreement (LoA)}

Overall, Pima showed a better performance with venous compared to capillary blood samples with a smaller range of bias and tighter LoA across studies with different predicate technologies. Studies reported absolute bias of Pima at CD4 $>500$ cells/ $\mu$ l ranging from -66.3 cells/ $\mu$ l (LoA: $-286.6,+154.0$ ) for venous [49] to -120.6 cells/ $\mu$ l (LoA: $-162.8,-78.4$ ) for capillary blood [46]. At lower CD4 ranges, bias were reported at +15 cells $/ \mu \mathrm{l}$ (LoA: -89 to +118 ) for CD4 $<200$ cell $/ \mu$ l using capillary blood [44]; and +5.1 cells $/ \mu$ l (LoA: $-126.6,+136.8)$ for CD4 $<350$ cell $/ \mu$ l using venous sample [49]. These data suggest that Pima overestimates the CD4 count at lower
CD4 ranges and underestimates the CD4 count at higher ranges; the bias was also increased at higher CD4 counts.

\section{Discussion}

Findings of this review suggest that POC CD4 testing can provide reliable results for making treatment decisions among HIV patients in LMICs. This review highlights the need for published data regarding the field evaluation of available POC CD4 tests, particularly in low-resource settings where these novel technologies are already demonstrating significant impact on the continuum of care for HIV-positive persons. Among six current or prospective commercially available POC CD4 technologies, only three have published studies that meet inclusion criteria and most of these used Pima as the index test. Among 19 studies reporting Pima performance data, 11 studies provided data required for meta-analysis of diagnostic test accuracy.

Findings on Pima performance from two studies using both venous and capillary blood showed that CD4 counts on venous blood samples produced more accurate results than capillary blood, with lower failure/error 


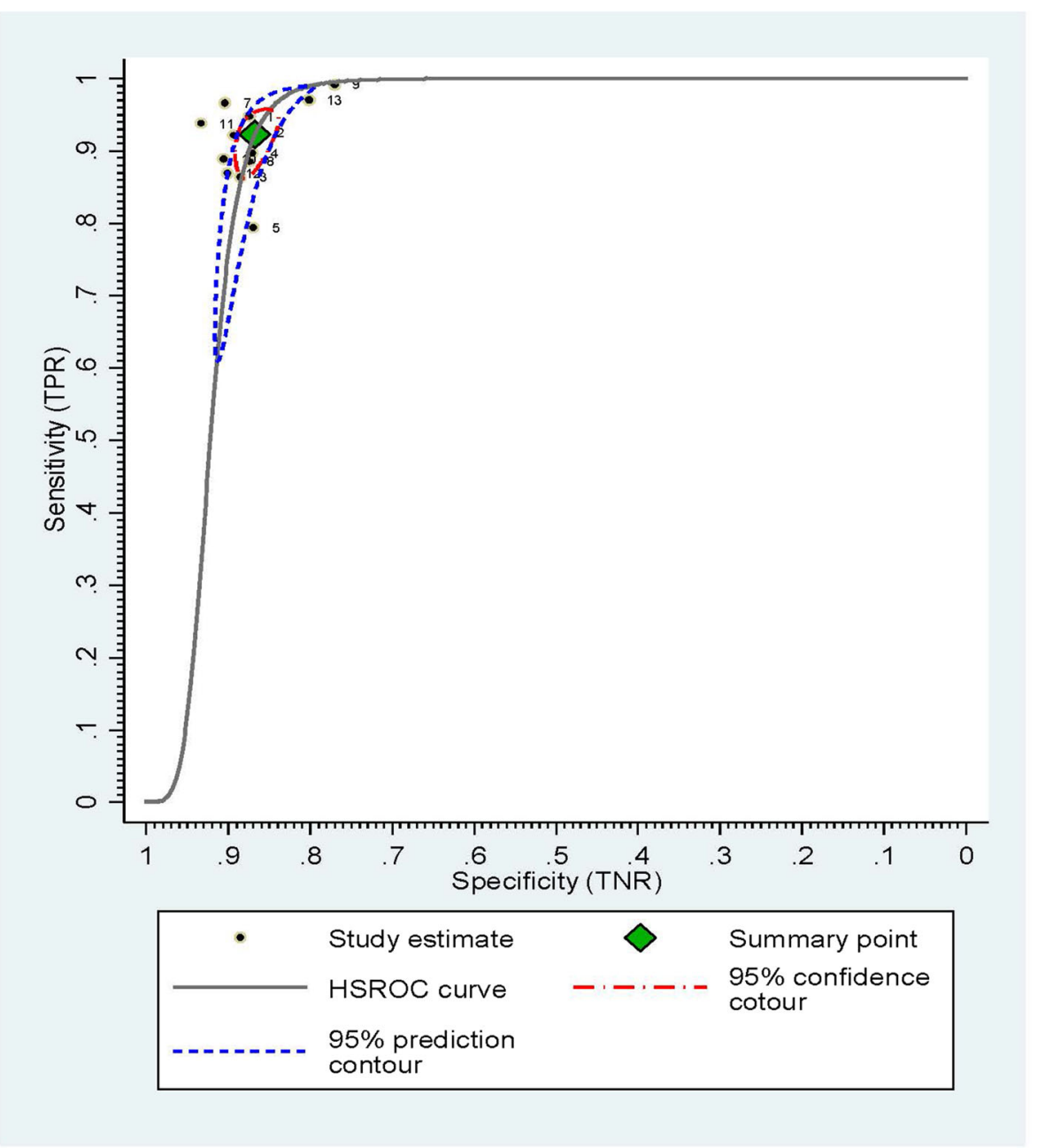

HSROC: Hierarchical summary receiver operating characteristics; TPR: True positive rate; TNR: True negative rate $¥$ : Data were pooled from 11 studies included in meta-analyses. Of those, two studies provided data on both venous and capillary blood leading to a total sample of 13 point estimates.

Fig. 3 HSROC curve from multi-level bivariate random effects model estimation of diagnostic performance of Pima at CD4 350 cells/ $\mu$ cut-off ${ }^{*}$ : plots observed sensitivity and specificity, diagnostic summary point, $95 \%$ confidence and prediction contours.

reading rate, the authors suggest that variation in test results was likely due to quality of capillary sampling [22, 44]. However, other evidence supports the use of either venous or capillary specimens $[35,40]$. Though not statistically significant, our meta-analysis shows that there is a trend towards a better performance of the test with venous blood, with a sensitivity of 0.94 for venous and 0.89 for capillary blood $(p=0.09)$ in identifying HIVpositive person eligible for ART at a cut-off of 350 cells/ $\mu \mathrm{L}$. If this is a true difference in performance of the test, the use of venous blood when using Pima for ART eligibility assessment would be preferable as it could reduce false negative test results which represent patient's missed opportunities for timely treatment initiation.

An observed wide range of failure rate of the Pima technology across studies is another attribute that needs further attention. Apart from technical and operational characteristics of the test, evidence from field studies suggests that performance of the test operator influences the accuracy of diagnostic test in the field [11, 22]. Therefore, the quality of training on POC testing for test operators and their supervisors becomes critically important to ensure effectiveness and efficiency of the technology in field settings. Of note, few of the included studies mentioned the effect of staff training on performance of POC CD4 test and none described details of the training program.

Bias in assessing the diagnostic accuracy of any new test could arise from faulty results of the reference test itself as there is no gold standard for CD4 testing although single platform flow cytometry has assumed that position. Thus evidence of participation and successful 
performance in external quality assurance (EQA) programs and performing duplicate tests on a sample using the predicate test are worthy recommendations in order to ensure the highest accuracy of predicate results. In this review only half of the included studies described EQA participation for the reference test and only one study conducted duplicate testing.

In order to better inform the decision making process on selection and adoption of POC CD4 testing in LMICs, further studies on currently and newly available POC CD4 technologies in various level settings and different geographic regions are needed. It is recommended that the quality of studies as well as quality of study reporting should be improved by following established standards $[26,54]$ and the focus of these future studies should not only be on test diagnostic accuracy but also on implementation aspects of the test, aiming at providing practical evidence to inform effective implementation strategies of POC CD4 testing.

There are two published systematic reviews and metaanalyses of the performance of POC CD4 tests, one by Scott et al. [19] and one by Peeling et al. [20]. In comparison, our review included 22 peer reviewed publications, providing a large increase in analysis of published work on POC CD4 technologies compared to the other reviews. Importantly, our study differed from the previously published reviews in that only studies conducted in field settings were included and thereby specially assessed field performance of the Pima. We demonstrated that misclassification by Pima, particularly with the use of capillary blood samples under field conditions, can be higher than that under a laboratory environment; thus our data vary with the earlier study where the reported probability of Pima misclassification was less than $10 \%$ [20]. A significant methodological strength of our meta-analysis is the direct estimation of the joint effect of blood sample type on Pima sensitivity and specificity simultaneously, using a bivariate multi-level random effect model with pooled study data. This is a significant improvement in statistical robustness compared to the simple comparison of $95 \%$ CI estimates for sensitivity and specificity applied in the other meta-analysis [19]. Encouragingly, our results, in line with findings from other reviews, confirm that POC CD4 tests also perform well if assessed specifically in field settings. Pima therefore has the potential for further deployment for ART eligibility assessment and treatment monitoring, especially in areas where laboratory-based CD4 testing is not available or difficult to access.

This review has some limitations which may affect the generalization of the findings. First, we included only published, peer-reviewed journal articles in English and this inclusion may overlook data from studies published in other languages or unpublished data from evaluations/ studies conducted by government agencies, reference facilities or similar institutions. The inclusion of conference abstracts has it strengths in limiting publication bias; however, confidence in these findings is limited as the quality of these studies has not been assessed via formal peer review. Second, only three of the six POC CD4 technologies found in this review were published with field study data and one technology (Pima) featured most prominently in the included studies. This presents challenges in terms of generalizing many of the findings of the review to "all" POC CD4 tests as it may be subjected to reporting bias. Third, interpretation of findings from meta-analyses of this review should be contextualized in terms of the limited diagnostic test data available from published studies. This limitation cannot be overcome until more data from field studies of different POC CD4 technologies, including the Pima, are available.

\section{Conclusions}

Findings of this review suggest that field studies of POC CD4 tests currently available on the market and those eagerly anticipated, conducted in LMICs where they are needed the most, remain much in need. The Pima ${ }^{\text {Ti }}$ CD4 showed acceptable diagnostic test accuracy using either venous or capillary blood. Existing evidence indicates that POC CD4 testing, can provide reliable results under field conditions and could play an important role in HIV continuum of care. This remains true, despite the changing landscape with respect to guidelines for ART initiation. Whilst evidence supports increasingly earlier commencement of treatment at an individual and community level, the financial reality is that in many parts of the world priority for ART initiation must still continue to be given to those with evidence of declining immune function. Further evidence is needed to ensure that efficacy is acceptable with both venous and capillary blood samples in field settings.

\section{Additional file}

Additional file 1: Annex 1. Medline Search Strategy - Descriptions of search terms used for literature search in Medline. (DOCX 16 kb)

\section{Abbreviations \\ ART: Antiretroviral therapy; DTA: Diagnostic test accuracy; \\ GLLAMM: Generalized latent and linear mixed modeling; HSROC: Hierarchical summary receiver operating characteristic; LMICs: Low and middle income countries; LoA: Limit of agreement; POC: Point-of-care; WHO: The World Health Organization}

Acknowledgments

The authors gratefully acknowledge the contribution to this work of the Victorian Operational Infrastructure Support Program received by the Burnet Institute. 


\section{Funding}

Funding was provided by the National Health and Medical Research Council of Australia (NHMRC) (Project grant GNT 1063725, and Career Development Fellowship to S. Luchters. Infrastructure for Research Institutes Support Scheme Grant. Suzanne Crowe is a recipient of an NHMRC Principle Research fellowship). Minh Pham received support via an International Postgraduate Research Scholarship (IPRS) from the Commonwealth of Australia and the Victorian International Research Scholarship (VIRS) from State Government of Victoria, Australia (VIRS). For the remaining authors none were declared.

\section{Availability of data and materials}

The dataset supporting the conclusion of this article is included within the article and its additional files.

\section{Authors' contributions}

ICMJE criteria for authorship read and met: MP, PA, LR, PG, DA, SC, SL. Developed review protocol: MP, SL, DA, SC. Performed literature search: LR, MP. Performed data extraction: MP, PG. Conducted Meta-analysis: PA, MP. Wrote the first draft of the manuscript: MP, LR, PA. Contributed to the writing of the manuscript: MP, LR, PA, SL, DA, SC. Agreed with the manuscript's results and conclusions: MP, PA, LR, PG, DA, SC, SL. All authors read and approved the final manuscript.

\section{Competing interest}

The authors declare that they have no competing interests.

\section{Consent for publication}

Not applicable.

\section{Ethics approval and consent to participate}

\section{Not applicable.}

\section{Author details}

${ }^{1}$ Burnet Institute, 85 Commercial Road, Melbourne, VIC 3004, Australia. ${ }^{2}$ Department of Epidemiology and Preventive Medicine, Faculty of Medicine Nursing and Health Science, Monash University, Melbourne, Australia. ${ }^{3}$ The Alfred Hospital, The lan Potter Library, Melbourne, VIC, Australia. ${ }^{4}$ Department of Immunology, Faculty of Medicine Nursing and Health Science, Monash University, Melbourne, Australia. ${ }^{5}$ Department of Infectious Diseases, The Alfred Hospital and Monash University, Melbourne, Australia. ${ }^{6}$ Department of Obstetrics and Gynecology, International Centre for Reproductive Health, Faculty of Medicine and Health Sciences, Ghent University, Ghent, Belgium.

Received: 24 June 2016 Accepted: 13 October 2016

Published online: 21 October 2016

\section{References}

1. UNAIDS. How AIDS changed Every thing: MD6: 15 years, 15 lessons of Hope from the AIDS response. 2015. Available at: http://www.unaids.org/en/ resources/documents/2015/MDG6_15years-15lessonsfromtheAIDSresponse.

2. TEMPRANO. A trial of early antiretrovirals and isoniazid preventive therapy in Africa. N Engl J Med. 2015;373:808-22.

3. INSIGHTSTART. Initiation of antiretroviral therapy in early asymptomatic HIV infection. N Engl J Med. 2015;373:795-807.

4. WHO. Guideline on when to start antiretroviral therapy and on pre-exposure prophylaxis for HIV. Geneva: World Health Organization; 2015. p. 78.

5. WHO. Consoldated guidlines on the use of antiretroviral drugs for treating and preventing HIV infection Recommendations for a public health approach. Geneva: World Health Organization; 2013. p. 272.

6. Ford $\mathrm{N}$, et al. The future role of CD4 cell count for monitoring antiretroviral therapy. Lancet Infect Dis. 2015;15(2):241-7.

7. Kranzer K, et al. Quantifying and addressing losses along the continuum of care for people living with HIV infection in sub-Saharan Africa: a systematic review. J Int AIDS Soc. 2012;15(2):17383.

8. Govindasamy D, Ford N, Kranzer K. Risk factors, barriers and facilitators for linkage to antiretroviral therapy care: a systematic review. AIDS. 2012; 26(16):2059-67.

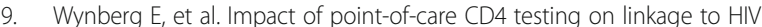
care: a systematic review. J Int AIDS Soc. 2014;17:18809.
10. Patten GE, et al. Impact on ART initiation of point-of-care CD4 testing at HIV diagnosis among HIV-positive youth in Khayelitsha, South Africa. J Int AIDS Soc. 2013;16:18518.

11. Mtapuri-Zinyowera S, et al. PIMA Point of Care CD4+ Cell Count Machines in Remote MNCH Settings: Lessons Learned from Seven Districts in Zimbabwe. Infect Dis (Auckl). 2013;6:51-60.

12. Feyissa G, Demissie T. Effect of point of care CD4 cell count tests on retention of patients and rates of antiretroviral therapy initiation in subSaharan African countries: a systematic review. JBI Database System Rev Implement Rep. 2014;12(4):395-429.

13. Faal $M$, et al. Providing immediate CD4 count results at HIV testing improves ART initiation. J Acquir Immune Defic Syndr. 2011;58(3):e54-9.

14. Jani IV, et al. Effect of point-of-care CD4 cell count tests on retention of patients and rates of antiretroviral therapy initiation in primary health clinics: an observational cohort study. Lancet. 2011:378(9802):1572-9.

15. van Rooyen $\mathrm{H}$, et al. High HIV testing uptake and linkage to care in a novel program of home-based HIV counseling and testing with facilitated referral in KwaZulu-Natal, South Africa. J Acquir Immune Defic Syndr. 2013;64(1):e1-8.

16. Zachariah $\mathrm{R}$, et al. Viewpoint: Why do we need a point-of-care CD4 test for low-income countries? Trop Med Int Health. 2011;16(1):37-41.

17. Hyle $\mathrm{E}$, et al. The Clinical and Economic Impact of Point-of-Care CD4 Testing in Mozambique and Other Resource-Limited Settings: A Cost-Effectiveness Analysis. PLoS Med. 2014;11(9), e1001725.

18. UNITAID. 2014 HIV/AIDS diagnostics technology landscape. 4th ed. 2014 Available at: http://www.unitaid.eu/en/resources/publications/technicalreports

19. Scott LE, et al. A meta-analysis of the performance of the Pima(TM) CD4 for point of care testing. BMC Med. 2015;13:168.

20. Peeling RW, et al. CD4 enumeration technologies: a systematic review of test performance for determining eligibility for antiretroviral therapy. PLoS One. 2015;10(3):e0115019.

21. Fajardo $\mathrm{E}$, et al. Errors generated by a point-of-care CD4+ T-lymphocyte analyser: a retrospective observational study in nine countries. Bull World Health Organ. 2015;93:623-30.

22. Glencross DK, et al. Performance evaluation of the Pima ${ }^{\mathrm{TM}}$ point-of-care CD4 analyser using capillary blood sampling in field tests in South Africa. J Int AIDS Soc. 2012;15(1):3.

23. Moher $\mathrm{D}$, et al. Preferred reporting items for systematic reviews and metaanalyses: the PRISMA statement. PLoS Med. 2009;6(7):e1000097.

24. Counsell $C$. Formulating questions and locating primary studies for inclusion in systematic reviews. Ann Intern Med. 1997;127(5):380-7.

25. Jackson $\mathrm{N}$, et al. Criteria for the systematic review of health promotion and public health interventions. Health Promot Int. 2005;20(4):367-74.

26. Whiting $P$, et al. The development of QUADAS: a tool for the quality assessment of studies of diagnostic accuracy included in systematic reviews. BMC Med Res Methodol. 2003:3:25.

27. Reitsma JB, et al. Bivariate analysis of sensitivity and specificity produces informative summary measures in diagnostic reviews. J Clin Epidemiol. 2005:58(10):982-90.

28. Huber PJ. The behavior of maximum likelihood estimates under nonstandard conditions. In: Fifth Berkeley Symposium on Mathematical Statistics and Probability. Berkeley: University of California Press; 1967. p. 221-33.

29. Dwamena BA. Midas: Stata module for Meta-analytical Integration of Diagnostic Accuracy Studies. 2007.

30. Rabe-Hesketh S, Pickles A, Taylor C. Generalized linear latent and mixed models. Stata Technical Bulletin 53. Stata Technical Bulletin Reprints. 2000;9: 293-307.

31. Harbord RM, Whiting P. metandi: Meta-analysis of diagnostic accuracy using hierarchical logistic regression. Stata Journal. 2009;9(2):211-29.

32. Rutter CM, Gatsonis CA. A hierarchical regression approach to meta-analysis of diagnostic test accuracy evaluations. Stat Med. 2001;20(19):2865-84.

33. Arnett N, Chang K, Schmitz M, Lemwayi R, Rwehumbiza P, Mwasekaga M. Healthcare workers' acceptance and performance of point-of-care CD4 testing in Dar es Salaam, Tanzania, 2011. In: 7th IAS Conference on HIV Pathogenesis and Treatment; Kuala Lumpur; 30 June-3 July 2013, 2013 (Abstract TUPDD0103 - Poster Discussion Session). 2013.

34. Rioja M, MbohKhan E, Nzuobontane D, Munyaburanga F, PiusMuffih T, Asong T. Improving access to care and treatment by strengthening CD4 count testing capacity in rural health districts in Cameroon through point-of-care technology. In: 7th IAS Conference on HIV 
Pathogenesis and Treatment; Kuala Lumpur; 30 June-3 July 2013., 2013 (Abstract WEPE609). 2013.

35. Zeh C, et al. Evaluation of Pima CD4 Point-Of-Care Device in Western Kenya for Potential Use in Field Settings. In: Conference on Retroviruses and Opportunistic Infections; Boston, Massachusetts; March 3-6 2014., (Abstract 624). 2014.

36. Thakar M, et al. Utility of the point of care CD4 analyzer, PIMA, to enumerate CD4 counts in the field settings in India. AIDS Res Ther. 2012;9(1):26.

37. Rathunde $L$, et al. Evaluation of the Alere Pima ${ }^{\text {TM }}$ for $C D 4+T$ lymphocytes counts in HIV-positive outpatients in Southern Brazil. Int J STD AIDS. 2014; 25:956-9.

38. Malagun $\mathrm{M}$, et al. Multisite evaluation of point of care CD4 testing in Papua New Guinea. PLoS One. 2014;9(11):e112173.

39. Jani IV, et al. Accurate CD4 T-cell enumeration and antiretroviral drug toxicity monitoring in primary healthcare clinics using point-of-care testing. AIDS. 2011:25(6):807-12.

40. Wade D, et al. WHO multicenter evaluation of FACSCount CD4 and Pima CD4 T-cell count systems: instrument performance and misclassification of HIV-infected patients. J Acquir Immune Defic Syndr. 2014;66(5):e98-107.

41. Gumbo P, et al. Analysis of Bias and ART Enrollment for a Point-of-Care CD4/CD4\% Analyzer. J AIDS Clin Res. 2013;4:247.

42. Wade D, et al. CD4 T-cell enumeration in a field setting: evaluation of CyFlow counter using the CD4 easy count kit-dry and Pima CD4 systems. PLoS One. 2013;8(9):e75484.

43. Picken $\mathrm{S}$, et al. The routine paediatric human immunodeficiency virus visit as an intervention opportunity for failed maternal care, and use of point-of-care CD4 testing as an adjunct in determining antiretroviral therapy eligibility. Southern African Journal of Epidemiology and Infection. 2014;29(2):70-4.

44. Diaw PA, et al. Multisite evaluation of a point-of-care instrument for CD4(+) T-cell enumeration using venous and finger-prick blood: the PIMA CD4. J Acquir Immune Defic Syndr. 2011;58(4):e103-11.

45. Myer $L$, et al. Comparison of point-of-care versus laboratory-based CD4 cell enumeration in HIV-positive pregnant women. J Int AIDS Soc. 2013;16(1):18649.

46. Manabe $Y C$, et al. Evaluation of portable point-of-care CD4 counter with high sensitivity for detecting patients eligible for antiretroviral therapy. PLoS One. 2012;7(4):e34319.

47. Bergeron $\mathrm{M}$, et al. Performance of the PointCare NOW system for CD4 counting in HIV patients based on five independent evaluations. PLoS One. 2012;7(8)::41166.

48. Mwau M, et al. Technical Performance Evaluation of the MyT4 Point of Care Technology for CD4+ T Cell Enumeration. PLoS One. 2014;9(9):e107410.

49. Galiwango RM, et al. Field evaluation of PIMA point-of-care CD4 testing in Rakai, Uganda. PLoS One. 2014;9(3):e88928.

50. Mtapuri-Zinyowera S, et al. Evaluation of the PIMA point-of-care CD4 analyzer in VCT clinics in Zimbabwe. J Acquir Immune Defic Syndr. 2010;55(1):1-7.

51. Mnyani CN, Mclntyre JA, Myer L. The reliability of point-of-care CD4 testing in identifying HIV-infected pregnant women eligible for antiretroviral therapy. J Acquir Immune Defic Syndr. 2012;60(3):260-4.

52. Gous N, et al. Feasibility of performing multiple point of care testing for HIV anti-retroviral treatment initiation and monitoring from multiple or single fingersticks. PLoS One. 2013;8(12):e85265.

53. Mwau M, et al. Evaluation of PIMA ${ }^{\text {TM }}$ point of care technology for CD4 T cell enumeration in Kenya. PLoS One. 2013;8(6):e67612.

54. Bossuyt PM, et al. Towards complete and accurate reporting of studies of diagnostic accuracy: The STARD Initiative. Ann Intern Med. 2003;138(1):40-4.

\section{Submit your next manuscript to BioMed Central and we will help you at every step:}

- We accept pre-submission inquiries

- Our selector tool helps you to find the most relevant journal

- We provide round the clock customer support

- Convenient online submission

- Thorough peer review

- Inclusion in PubMed and all major indexing services

- Maximum visibility for your research

Submit your manuscript at www.biomedcentral.com/submit 